\title{
Bypass gástrico como modelo de manejo metabólico en la diabetes mellitus tipo 2 y obesidad grado 1, en comparación con el tratamiento convencional
}

\author{
Gastric bypass as a metabolic management model in type 2 diabetes mellitus \\ and obesity grade 1, compared to conventional treatment
}

Luis Guillermo Menchaca Ramos, * María del Carmen Larios Forte,** Roberto Alain Garza Muñoz***

Palabras clave:

Bypass gástrico, cirugía metabólica, obesidad, diabetes.

Key words: Gastric bypass, metabolic surgery, obesity, diabetes.

\begin{abstract}
Abreviaturas:
$\mathrm{DM}=$ Diabetes mellitus. IMC = Índice de masa corporal.

ENSANUT $=$ Encuesta Nacional de Salud y Nutrición de Medio Camino 2016.

HbA1c = Hemoglobina glicosilada.

GLP1 = Péptido similar al glucagón 1 . GIP = Polipéptido inhibidor gástrico. YY = Péptido YY. DPP4 = Dipeptidil peptidasa 4. $\mathrm{OR}=$ Odds ratio. $\mathrm{IC}=$ Índice de confianza.

* Cirujano General, adscrito al Servicio de Cirugía General.

** Radióloga, jefa del Departamento de Investigación y de Radiología e Imagen. *** Endocrinólogo, adscrito al Servicio de Medicina Interna.
\end{abstract}

Hospital Regional de Alta Especialidad, ISSSTE, Monterrey.

\section{RESUMEN}

Antecedentes: La DM2 es un problema de salud pública mundial. En 1994 Pories describió la corrección de la glucemia plasmática en pacientes obesos con DM postbypass. Rubino, Scopinaro (2002) y Carbajo (2008) confirmaron estos datos desde la primera semana del postoperatorio. Observaciones en pacientes sometidos a derivaciones gastroyeyunales por enfermedad ácido péptica o neoplasias reportan resultados semejantes. La Asociación Americana de Diabetes (ADA) justifica que bajo protocolos de investigación se realice este procedimiento de bypass gástrico en pacientes con IMC entre 30 y 35. Material y métodos: Es un estudio experimental no ciego, con una cohorte de 47 pacientes de DM2 y obesidad grado 1 del HRAE Monterrey, ISSSTE, donde se realizó bypass gástrico a 13 pacientes (casos) para compararse con aquéllos en tratamiento clínico convencional (controles) por un año de seguimiento. Resultados: Los 13 pacientes intervenidos (ocho mujeres y cinco hombres) iniciaron con una media de $\mathrm{HbAl} \mathrm{c}=9.7 \%$, que disminuyó a $6 \%$ al tercer mes postquirúrgico; sólo tres continúan con hipoglucemiantes orales a menor dosis. De los 34 pacientes control, la media inicial de HbA1c2015 = 9.2\%, y en 2016, media $=8.9 \%$. Discusión: La DM2 y obesidad 1 siguen siendo un reto difícil de controlar. El bypass gástrico demostró ser efectiva en el $69 \%$ de los casos operados, logrando control de los niveles $\mathrm{HbA} 1 \mathrm{c}<6$. Conclusiones: Este grupo de pacientes con DM2 e IMC $<35$ y > 30 es altamente vulnerable, por lo que debería incluirse en los procedimientos quirúrgicos bariátricos y evitar las complicaciones con daño irreparable.

\section{ABSTRACT}

Background: Type 2 diabetes (DM2) is a public health problem worldwide. In 1994 Pories reported glycemic correction after gastric bypass in obese patients with DM2; then, Rubino, Scopinaro (2002) and Carbajo (2008) confirmed this glycemic normalization during the first postoperative week. Studies in patients submitted to gastrojejunal surgery due to peptic acid disease or neoplasms reported similar results in blood glucose control. The American Diabetes Association justifies this procedure to be performed under protocol study in patients with BMI between 30 and 35. Material and methods: A non-blind experimental cohort study; 47 patients with type 2 diabetes and grade I obesity were selected at the High Specialty Regional Monterrey Hospital, ISSSTE; 13 patients (cases) underwent gastric bypass and were compared versus 34 patients in conventional clinical treatment (control) in one-year follow-up. Results: The 13 patients who underwent surgery (eight female and five male) started with a mean $\mathrm{HbA1c}=9.7 \%$, which decreased to $6 \%$ post-surgery. Only three of them continue with oral hypoglycemic agents in minimal doses. Regarding the 34 clinical treatment patients, their initial (2015) mean $\mathrm{HbA1c}=9.2 \%$, and their 2016 mean $=8.9 \%$, maintaining medications. Discussion: The control group had a high risk of complications. Gastric bypass showed to be more effective in the control of $\mathrm{HbAlc}$. Conclusions: Patients with DM2 and BMI between 30 and 35 are a highly vulnerable group, which should be included in bariatric surgical procedures to avoid complications with irreparable damage.

\section{INTRODUCCIÓN}

T a diabetes mellitus tipo 2 (DM2) es un ـimportante problema de salud pública mundial que afecta a más de 150 millones de personas actualmente y se duplicará para el año 2025. ${ }^{1,2}$ En México, la Encuesta Nacional del año $2016^{3}$ reportó que a $9.4 \%$ de la población se le ha diagnosticado DM2. Por ello, la meta urgente del tratamiento hoy por hoy es llevar a 
cabo medidas de prevención y control de esta epidemia y tratar de evitar complicaciones por sus riesgos cardiovasculares, falla renal, retinopatía, amputaciones por insuficiencia vascular o fallecimiento prematuro. ${ }^{1-5}$

De acuerdo con los criterios establecidos por la Asociación Americana de Diabetes (ADA 2016), ${ }^{5}$ el diagnóstico se basa en las siguientes mediciones: glucosa preprandial mayor de $126 \mathrm{mg} / \mathrm{dl}$, postprandial mayor de $200 \mathrm{mg} /$ $\mathrm{dl}$, hemoglobina glicosilada mayor del $6.5 \%$ y glucemia al azar mayor de 200 mg/dl, con síntomas clásicos; las terapias actuales como dieta, ejercicio, terapia conductual, hipoglucemiantes orales e insulina mantienen con dificultad el estado euglucémico del paciente. ${ }^{4-7}$

El impacto por los procedimientos quirúrgicos bariátricos del tipo bypass convencional en el control metabólico observado de los pacientes diabéticos descritos por el Dr. Pories ${ }^{8,9}$ y sus colaboradores, así como el procedimiento de Scopinaro ${ }^{10}$ y el de una sola anastomosis detallado por los doctores Carbajo ${ }^{11}$ y Cerón ${ }^{12}$ reportan la normalización de los niveles de glucosa plasmática a los pocos días del postoperatorio; se sugiere que esto puede ser consecuencia de evitar el paso de los alimentos a través del antro gástrico, duodeno y yeyuno proximal, y la alteración de señales hormonales que ocurren tanto en el yeyuno como en el íleon. ${ }^{13-17} \mathrm{El}$ seguimiento a corto plazo y por más de 20 años de estos pacientes no ha reportado las complicaciones propias de la DM2. ${ }^{18-21}$

De la modificación anatómica gastrointestinal por el bypass gástrico se derivan dos hipótesis en diferentes niveles de acción: del intestino proximal y del intestino distal. ${ }^{22-25} \mathrm{La}$ hipótesis del intestino proximal sugiere que la exclusión del paso del alimento a través del duodeno y yeyuno proximal deriva en una mejoría del control glucémico, mientras la hipótesis del intestino distal sugiere que el alimento parcialmente digerido que llega de manera temprana al íleon distal contribuye al estímulo para la producción de GLP1 y péptido YY, que favorecen a la mejoría en la secreción y acción de la insulina. Con ello, se considera como mecanismo de acción a la estimulación hormonal intestinal, específicamente GLP1 y GIP. Todos estos estímulos de las hormonas intestinales favorecen una mejor secreción y acción temprana de la insulina y la incitación favorable sobre las células pancreáticas. ${ }^{13-17}$

Rubino y su grupo (2008), al explicar estos cambios, proponen la teoría de las incretinas y antiincretinas, en donde se explica que el efecto incretínico de los péptidos gastrointestinales (GIP, YY, los inhibidores de la DPP4 y otros) favorece el trabajo de la insulina, el ingreso de la glucosa al interior de las células y evita la apoptosis celular pancreática, versus el efecto antiincretínico de las células que se encuentran en el duodeno y primer segmento del yeyuno, que al ser estimuladas por el paso del alimento inician la cascada del bloqueo a la insulina, la resistencia a la misma y, finalmente, la apoptosis celular de las células beta del páncreas en pacientes que tienen los genes que predisponen al desarrollo de la DM2. ${ }^{13-17}$ Estos hallazgos sugieren un potencial efecto del intestino proximal en la patogénesis de la enfermedad y apuntan hacia nuevas alternativas terapéuticas para el manejo de la DM2. ${ }^{13,14}$

Ninguna intervención quirúrgica abdominal está exenta de complicaciones; se ha descrito una morbilidad de $8-12 \%$ en procedimientos similares y mortalidad menor a $1 \%$; al recordar que la morbimortalidad de la diabetes es de 4.5 a $8 \%$ por año en el paciente obeso que no se opera, ${ }^{25-30}$ la balanza se inclina favorablemente hacia el procedimiento quirúrgico que, con bases científicas, estamos proponiendo.

Por otro lado, el bypass gástrico, según la Norma Oficial Mexicana NOM-008SSA3-2010 31 en relación con la obesidad, podrá realizarse en pacientes con un índice de masa corporal (IMC) de 35 asociado a comorbilidad o mayor de 40 sin comorbilidades. La Asociación Americana de la Diabetes ${ }^{4}$ reporta que aquellos pacientes diabéticos tipo 2 con IMC entre 30 y 35 podrán ser sometidos a bypass gástrico sólo bajo protocolos de investigación; basándonos en esta condición, nuestra investigación incluye a este grupo de pacientes.

Hay referencias en modelos animales diabéticos no obesos, así como en algunas publicaciones, de la realización del procedimiento bariátrico en pacientes con IMC menor de 35 y mayor de 30; $;^{32-35}$ algunas lo comparan con tratamiento médico, pero se requiere de un 
mayor número de estudios para una mejor evidencia.

\section{MATERIAL Y MÉTODOS}

Fue un estudio experimental piloto tipo casos y controles anidado en una cohorte de pacientes diabéticos DM2 del Hospital Regional de Alta Especialidad de Monterrey, ISSSTE, de marzo de 2015 a junio de 2016, no ciego, ambispectivo y comparativo, de 47 pacientes: el grupo de casos $(n=13)$ fueron pacientes que por consentimiento informado y aceptado fueron sometidos a bypass gástrico (por cualquiera de los dos métodos, Y de Roux o Bagua) y el grupo de controles $n=34$ fueron aquéllos en tratamiento clínico convencional. Se compararon las variables demográficas y las cuantitativas (niveles séricos de glucosa e IMC) de ambos grupos por análisis estadístico de $\chi^{2}$ y t Student, cálculo de significancia estadística y OR con IC 95\%. El protocolo fue validado y autorizado por los comités de investigación y de ética del Hospital Regional de Alta Especialidad de Monterrey, ISSSTE, y por sus correspondientes a nivel central de la Ciudad de México, del ISSSTE.

\section{Tuvo dos fases:}

Fase I (run-in). En esta fase se invitó a participar a pacientes diabéticos tipo 2 con IMC mayor de 30 y menor a 35 que firmaron la carta de consentimiento informado con las indicaciones correspondientes al tratamiento médico de DM2 para su participación por tres meses; mientras tanto, se solicitaron los exámenes de laboratorio básicos: $\mathrm{BH}, \mathrm{QS}, \mathrm{PFH}, \mathrm{TP}$, valoración por endocrinología, psicología, nutrición y neumología.

Fase II. Asignación del grupo casos: $\mathbf{n}=\mathbf{1 3}$ pacientes que cumplieron con las indicaciones de la fase I, a los que se les invitó y aceptaron participar en el procedimiento quirúrgico (bypass gástrico) para evaluar su control metabólico; y asignación del grupo controles: $\mathbf{n}=$ 34 pacientes que cumplieron los exámenes y evaluaciones, y que no aceptaron por el momento el procedimiento quirúrgico (Figura 1), a los que se les dio seguimiento bajo el tratamiento médico convencional.

\section{Criterios de inclusión}

- Ambos sexos.

- Edad de 25 a 60 años.

- Diabéticos tipo 2 diagnosticados de acuerdo con los criterios ADA 2016.

- Derechohabientes del ISSSTE.

- Con seguimiento dentro del Hospital Regional ISSSTE de Alta Especialidad de Monterrey.

- Con tratamiento médico documentado en el expediente.

- Con IMC > 30 y < 35 al momento de aceptar participar en el estudio.

- Valoración psicológica favorable.

- Valoración por endocrinología, neumología, nutrición y medicina interna.

- Con posibilidad de realizar ejercicio físico.

- Sin cirugías previas en el estómago o duodeno.

- Con probabilidad de vida de más de dos años al momento de su selección.

- En el caso de mujeres, prueba de embarazo negativa al momento de su selección.

- Firma de la carta de consentimiento informado.

- Contar con por lo menos un cuidador primario.

- Posibilidad de seguimiento periódico en los siguientes dos años posteriores a la intervención.

\section{Criterios de exclusión}

- Enfermedad psiquiátrica.

- Infarto antiguo del miocardio u otras cardiopatías.

- Embarazo al momento de la intervención.

- Antecedentes de neoplasias malignas previas que pudieran poner en riesgo la evolución o acortar prematuramente el periodo de vida del paciente.

- Inmunodeficiencia adquirida (HIV).

- Hepatopatías y padecer enfermedad neoplásica maligna.

- Cirugías bariátricas previas.

- Etilismo.

- Toxicomanías.

- Con insuficiencia renal crónica.

\section{Criterios de eliminación}

- Que no acudieran a sus valoraciones de niveles séricos completas. 
Bypass gástrico para el control metabólico en diabetes y obesidad con IMC $>30<35$

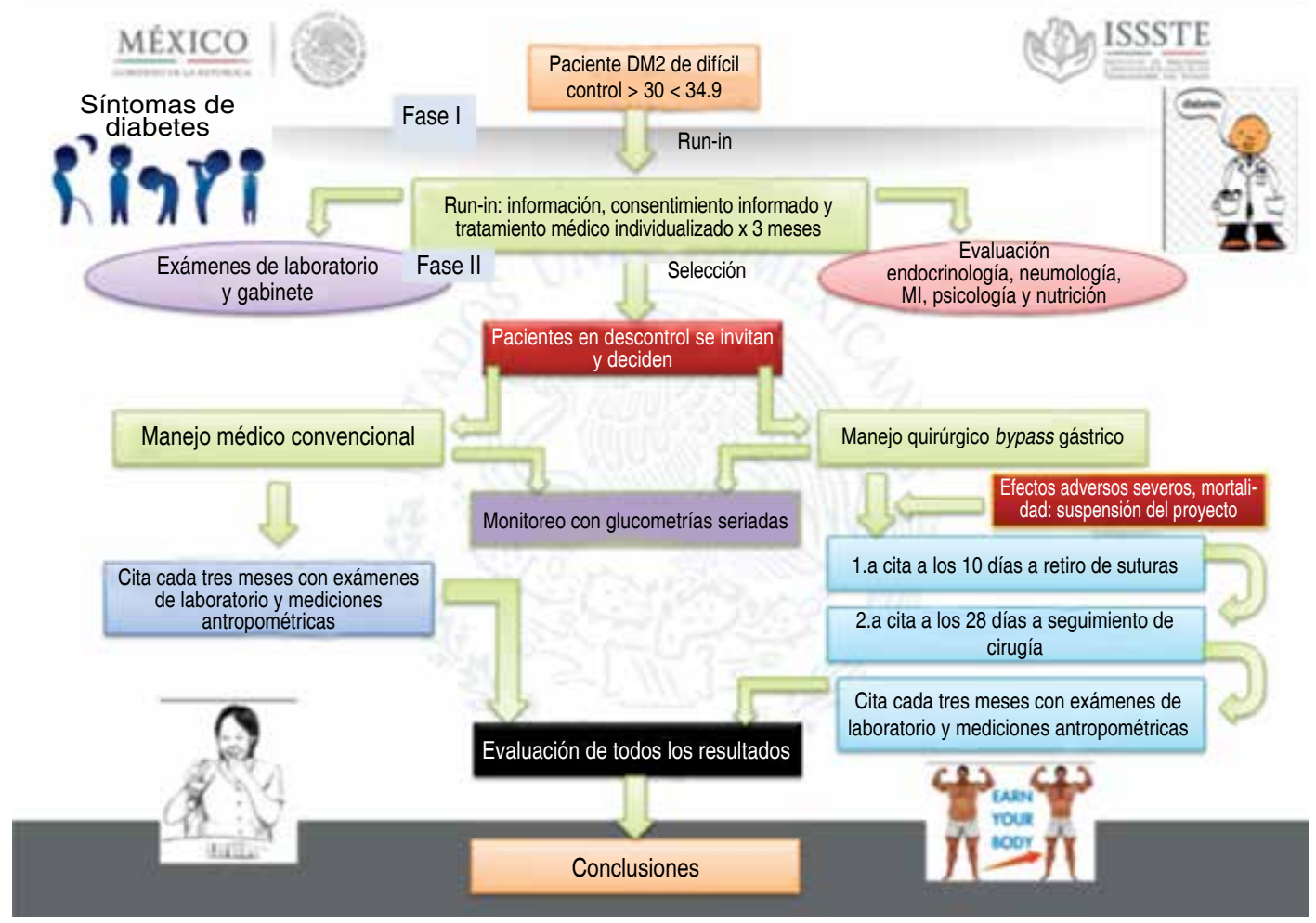

Figura 1. Algoritmo de selección.

- Cambio de domicilio fuera de la región por criterio del propio paciente.

- Que durante el estudio desarrollaran alguna enfermedad terminal.

- Que en caso de complicaciones que sobrepasaran los reportes internacionales debieran ser sometidos a la opinión técnica del comité de ética e investigación para evaluar la continuidad o no del protocolo.

\section{Análisis estadístico}

Primero se realizó análisis descriptivo (frecuencias) del comportamiento de los 34 pacientes con DM2 e IMC > $30<35$. Posteriormente, se compararon las diferentes características entre ambos grupos (casos y controles) de respuesta de acuerdo al sexo, edad y peso; para ello, se utilizó la prueba de $\chi^{2}$ con cálculo de significancia estadística ( $p \leq 0.05)$, así como cálculo de la correlación por edad con prueba t Student ( $p \leq 0.05)$; posteriormente, se compararon las respuestas de la $\mathrm{HbA} 1 \mathrm{c} \%$ y del IMC tras el tratamiento quirúrgico y médico convencional entre los casos $(n=13)$ y los controles $(n=$ 34), con cálculo de su significancia estadística, determinación del OR e IC 95\%

\section{RESULTADOS}

En el Hospital Regional de Alta Especialidad de Monterrey, ISSSTE, se realizó este estudio en 47 pacientes con DM2 y obesidad grado 1, 28 del sexo femenino y 19 del masculino, entre los 28 y 60 años de edad. Después de haber cumplido con la fase 1 y 2 del run-in, se llevó a cabo bypass gástrico en 13 pacientes (ocho mujeres y cinco hombres, media de edad $=$ 47.3 años), y manejo médico convencional en 34 (20 mujeres y 14 hombres, media de edad $=54.1$ años).

- Se encontró que ambos grupos fueron similares en cuanto a sexo, edad y peso (IMC), sin diferencia estadística significativa entre ellos (Cuadros I a IV de frecuencias), $\mathrm{p}=$ 0.6 . 
Cuadro I. Grupo quirúrgico por sexo.

\begin{tabular}{|c|c|c|c|}
\hline \multicolumn{2}{|c|}{ Bypass } & \multirow[b]{2}{*}{ Frecuencia } & \multirow[b]{2}{*}{ Porcentaje } \\
\hline Casos & Género & & \\
\hline \multirow[t]{3}{*}{ Válido } & Femenino & 8 & 61.5 \\
\hline & Masculino & 5 & 38.5 \\
\hline & Total & 13 & 100.0 \\
\hline
\end{tabular}

\section{Cuadro II. Grupo control por sexo.}

\begin{tabular}{llcc} 
Control & Género & Frecuencia & Porcentaje \\
\hline \multirow{2}{*}{ Válido } & Femenino & 20 & 58.8 \\
& Masculino & 14 & 41.2 \\
& Total & 34 & 100.0
\end{tabular}

Cuadro III. Distribución por edades en ambos grupos.

\begin{tabular}{lccccc} 
Edad en años & N & Mínimo & Máximo & Media & $\begin{array}{c}\text { Desviación } \\
\text { estándar }\end{array}$ \\
\hline Caso Precirugía & 13 & 29 & 60 & 47.3 & 10.7 \\
Control 2015 & 34 & 31 & 60 & 54.1 & 7.2
\end{tabular}

Cuadro IV. IMC de los casos y controles iniciales.

\begin{tabular}{lccccc}
$\begin{array}{l}\text { Índice de masa } \\
\text { corporal (IMC) }\end{array}$ & $\mathrm{N}$ & Mínimo & Máximo & Media & $\begin{array}{c}\text { Desviación } \\
\text { estándar }\end{array}$ \\
\hline Caso Precirugía & 13 & 30.0 & 34.9 & 32.8 & 1.6 \\
Control 2015 & 34 & 27.4 & 36.9 & 32.6 & 1.8
\end{tabular}

- Al compararlos en relación con la HbA1c (media) de los casos pre- y postcirugía versus los controles medidos del 2015 al 2016 , se observó una diferencia significativa de $\mathrm{p}=0.043$, con un riesgo relativo positivo $\mathrm{OR}=1.6$, e IC $95 \%=1.0-2.4$ a favor del bypass gástrico.

- El grupo controles: en sus niveles de HbA1c\% sólo mostró cambios leves: disminuyó 0.3 puntos porcentuales en el seguimiento de un año; la media inicial fue $=9.2 \%$ y la media final $=8.9 \%$. Asimismo, se observó en forma individual (por cada control) que hubo una mayor labilidad en la estabilidad de la HbA1c, con fluctuaciones de los niveles durante todo el seguimiento (12 meses), con solo un paciente que logró disminuir su HbA1c adecuadamente.

- El grupo casos: disminuyó 3.7 puntos porcentuales tras la cirugía (de $\mathrm{m}=9.7 \%$ a $\mathrm{m}=6.0 \%$, respectivamente). En la actualidad, de los 13 pacientes, 10 no requieren tratamiento médico convencional y tres disminuyeron sus dosis de hipoglucemiantes orales; ninguno utiliza insulina y mantienen una $\mathrm{HbA} 1 \mathrm{c}$ en promedio de 6\% (Cuadro V).

Complicación: se presentó una complicación con una fuga intestinal, la cual fue resuelta y el paciente egresado. No hubo mortalidad por los procedimientos.

- En relación con el IMC, en el grupo controles, la media inicial $=32.6$ y después de un año de seguimiento, la media $=$ 32.4; así mismo, en el grupo casos, la $\mathrm{m}=$ 32.8 bajó a $\mathrm{m}=28.7$ (pre- y postcirugía, respectivamente, $p=0.7$ ), con una diferencia estadística no significativa entre ambos grupos de $p=0.27, \mathrm{OR}=1.1$ e IC $95 \%$ $=0.54-2.21$ (Cuadro VI).

\section{DISCUSIÓN}

- El manejo convencional de la DM2 con obesidad grado 1 sigue siendo un reto y es difícil obtener resultados adecuados.

- Los pacientes del grupo control persistieron con cifras elevadas de HbA1c, lo que los llevará a un deterioro sistémico progresivo en un plazo relativamente corto, con la morbimortalidad asociada.

- El bypass gástrico demostró ser efectiva en más del 50\% de los casos operados (69\%) a partir de la primera semana postcirugía al lograr un control adecuado de los niveles de glucemia en ayuno, y al tercer mes, niveles de $\mathrm{HbA} 1 \mathrm{c}$ en promedio de $6 \%$ (lo que disminuirá considerablemente el riesgo de complicaciones en estos pacientes. 


\begin{tabular}{|c|c|c|c|c|c|}
\hline Caso Hb Glic./IMC & $\mathrm{N}$ & Mínimo & Máximo & Media & Desviación estándar \\
\hline $\mathrm{Hb}$ Glic. Precirugía caso & 13 & 6.1 & 14.0 & 9.7 & 2.6 \\
\hline Hb Glic. Postbypass caso & 13 & 4.3 & 8.5 & 6.0 & 1.2 \\
\hline IMC Precirugía caso & 13 & 30.0 & 34.9 & 32.8 & 1.6 \\
\hline IMC Postbypass caso & 13 & 25.0 & 34.0 & 28.7 & 2.4 \\
\hline N válido (por lista) & 13 & & & & \\
\hline
\end{tabular}

Cuadro VI. IMC y HbA1c del grupo control antes y después del tratamiento médico convencional.

\begin{tabular}{lrrrrc}
$\begin{array}{c}\text { Control Hb Glic/IMC } \\
\text { 2015-16 }\end{array}$ & N & Mínimo & Máximo & Media & Desviación estándar \\
\hline Control Hb Glic. 2015 & 34 & 5.3 & 14.0 & 9.2 & 2.1 \\
Control Hb Glic. 2016 & 34 & 5.5 & 13.8 & 8.9 & 1.9 \\
Control IMC 2015 & 34 & 27.4 & 36.9 & 32.6 & 1.8 \\
Control IMC 2016 & 34 & 26.9 & 37.2 & 32.4 & 1.9
\end{tabular}

- El bypass gástrico permite abrir una ventana en el tiempo al retrasar la aparición de complicaciones y tener una nueva oportunidad de llevar una mejor calidad de vida, además de ayudar en un mejor apego al tratamiento y seguimiento.

- Es necesario aumentar el número de pacientes para confirmar la significancia estadística y elevar la tendencia en la mejoría de los niveles séricos de la HbA1c según los resultados obtenidos en este estudio piloto.

\section{CONCLUSIONES}

El presente estudio piloto demuestra que:

- El bypass gástrico muestra una tendencia clara y objetiva para disminuir los niveles elevados de $\mathrm{HbA} 1 \mathrm{c}$ de las personas con DM2 e IMC $>30$ y $<35$ (grado 1 de obesidad), controlando eficientemente la DM2, al compararlas con los pacientes que reciben el tratamiento médico convencional.

- El bypass gástrico, en forma casi inmediata a la cirugía (una semana después), logra disminuir los niveles de glucosa sérica y lleva a la normalidad a los pacientes con DM2, quienes dejan de ingerir hipoglucemiantes en el $69 \%$ de los casos.

- Las personas con DM2 sin un control adecuado e IMC $>30$ y $<$ de 35 son un grupo de pacientes altamente vulnerable que desarrollará complicaciones desastrosas en un periodo relativamente corto.

- Se debe considerar proponer esta alternativa quirúrgica para lograr el manejo oportuno del control metabólico de estos pacientes y la disminución en un tiempo relativamente corto del riesgo de las temidas complicaciones; de este modo, ofrecerles una vida de mejor calidad y más duradera.

\section{Agradecimientos}

Srita. Martha Cepeda García por su gran apoyo y trabajo incondicional; Dr. Saúl Humberto Méndez Luna, Cirujano General; Dr. Francisco Castillo Sánchez, Neumólogo; Dr. José Bocanegra López, Medicina Interna; Lic. Juan Manuel Covarrubias, Psicólogo; Lic. Felícitas Cuéllar, Nutrióloga, y Dra. Nora Elia Gutiérrez Tijerina, Medicina Familiar, por su eficiente cooperación y atención como interconsultantes. 


\section{REFERENCIAS}

1. King $H$, Aubert RE, Herman WH. Global burden of diabetes, 1995-2025: prevalence, numerical estimates, and projections. Diabetes Care. 1998; 21: 1414-1431.

2. Aguilar-Salinas CA, Velazquez-Monroy O, GómezPérez FJ, Gonzalez-Chávez A, Esqueda AL, MolinaCuevas $V$ et al. Characteristics of patients with type 2 diabetes in Mexico: results from a large populationbased nationwide survey. Diabetes Care. 2003; 26: 2021-2026.

3. Encuesta Nacional de Salud y Nutrición de Medio Camino 2016 (ENSANUT 2016). Instituto Nacional de Salud Pública. p. 46. Disponible en: http://www. epidemiologia.salud.gob.mx/doctos/encuestas/ resultados/ENSANUT.pdf

4. American Diabetes Association (ADA). 2017. Disponible en: http://professional.diabetes.org/sites/ professional.diabetes.org/files/media/dc_40_s1_final. pdf

5. American Diabetes Association. Standards of medical care in diabetes 2010. Diabetes Care. 2010; 33 Suppl 1: S11-S61.

6. Unger J. Diagnosis and management of type 2 diabetes and prediabetes. Prim Care. 2007; 34: 731-59, vi.

7. Alberti KG, Zimmet PZ. Definition, diagnosis and classification of diabetes mellitus and its complications. Part 1: Diagnosis and classification of diabetes mellitus, provisional report of a WHO consultation. Diabet Med. 1998; 15: 539-553.

8. Pories WJ, Swanson MS, MacDonald KG, Long SB, Morris PG, Brown BM, et al. Who would have thought it? An operation proves to be the most effective therapy for adult-onset diabetes mellitus. Ann Surg. 1995; 222: 339-350; discussion 350-352.

9. Pories WJ. Diabetes: the evolution of a new paradigm. Ann Surg. 2004; 239: 12-13.

10. Scopinaro N, Papadia F, Camerini G, Marinari G, Civalleri D, Gian Franco A. A comparison of a personal series of biliopancreatic diversion and literature data on gastric bypass help to explain the mechanisms of resolution of type 2 diabetes by the two operations. Obes Surg. 2008; 18: 1035-1038.

11. Carbajo MA, Ortiz de SJ, García-Lanza C, Pérez MM Fonseca OG, Castro MJ. Bypass gástrico laparoscópico de una sola anastomosis (BAGUA) asistido con brazo robótico: Técnica y resultados en 1,126 pacientes. Rev Mex Cir Endoscop. 2008; 9: 6-13.

12. Cerón-Rodríguez F, Márquez-González SM, AmpudiaChávez JD, Zúñiga-García Y, Valdés-Sánchez AP, Zubirán-Moreno JM, et al. Bypass gástrico López Mateos. Descripción de la técnica. Rev Mex Cir Endoscop. 2014; 15: 6-9.

13. Rubino F, Forgione A, Cummings DE, Vix M, Gnuli D Mingrone $\mathrm{G}$, et al. The mechanism of diabetes control after gastrointestinal bypass surgery reveals a role of the proximal small intestine in the pathophysiology of type 2 diabetes. Ann Surg. 2006; 244: 741-749.

14. Rubino F, Gagner M. Potential of surgery for curing type 2 diabetes mellitus. Ann Surg. 2002; 236: 554-559.

15. Rubino F, Gagner M, Gentileschi P, Kini S, Fukuyama $\mathrm{S}$, Feng J, et al. The early effect of the Roux-en-Y gastric bypass on hormones involved in body weight regulation and glucose metabolism. Ann Surg. 2004; 240: 236-242.

16. Pinheiro JS, Schiavon CA, Pereira PB, Correa JL, Noujaim P, Cohen R. Long-long limb Roux-en-Y gastric bypass is more efficacious in treatment of type 2 diabetes and lipid disorders in super-obese patients. Surg Obes Relat Dis. 2008; 4: 521-525; discussion 526-527.

17. Rubino F. Is type 2 diabetes an operable intestinal disease? A provocative yet reasonable hypothesis. Diabetes Care. 2008; 31 Suppl 2: S290-S296.

18. Batsis JA, Romero-Corral A, Collazo-Clavell ML, Sarr MG, Somers VK, Lopez-Jimenez F. Effect of bariatric surgery on the metabolic syndrome: a populationbased, long-term controlled study. Mayo Clin Proc. 2008; 83: 897-907.

19. Pories WJ. Bariatric surgery: risks and rewards. J Clin Endocrinol Metab. 2008; 93: S89-S96.

20. Franz MJ, VanWormer JJ, Crain AL, Boucher JL, Histon T, Caplan W et al. Weight-loss outcomes: a systematic review and meta-analysis of weight-loss clinical trials with a minimum 1-year follow-up. J Am Diet Assoc. 2007; 107: 1755-1767.

21. Jayasena $\mathrm{CN}$, Bloom SR. Role of gut hormones in obesity. Endocrinol Metab Clin North Am. 2008; 37: 769-787, xi.

22. Hickey MS, Pories WJ, MacDonald KG Jr, Cory KA, Dohm GL, Swanson MS, et al. A new paradigm for type 2 diabetes mellitus: could it be a disease of the foregut? Ann Surg. 1998; 227: 637-643; discussion 643-644.

23. Moo TA, Rubino F. Gastrointestinal surgery as treatment for type 2 diabetes. Curr Opin Endocrinol Diabetes Obes. 2008; 15: 153-158.

24. Buchwald H, Estok R, Fahrbach K, Banel D, Jensen $\mathrm{MD}$, Pories WJ, et al. Weight and type 2 diabetes after bariatric surgery: systematic review and meta-analysis. Am J Med. 2009; 122: 248-256.e5.

25. Thaler JP, Cummings DE. Minireview: Hormonal and metabolic mechanisms of diabetes remission after gastrointestinal surgery. Endocrinology. 2009; 150: 2518-2525.

26. MacDonald KG Jr, Long SD, Swanson MS, Brown BM, Morris P, Dohm GL, et al. The gastric bypass operation reduces the progression and mortality of non-insulindependent diabetes mellitus. J Gastrointest Surg. 1997; 1: 213-220; discussion 220.

27. Cummings DE. Endocrine mechanisms mediating remission of diabetes after gastric bypass surgery. Int J Obes (Lond). 2009; 33 Suppl 1: S33-S40.

28. Folli F, Pontiroli AE, Schwesinger WH. Metabolic aspects of bariatric surgery. Med Clin North Am. 2007; 91: 393-414.

29. Paula-Meyer M. Gastric bypass surgery patients at high risk of complications. Medscape. 2003.

30. Dixon J. Survival advantage with bariatric surgery: Report from the 10th International Congress on Obesity. Surg Obes Relat Dis. 2006; 2: 585-586.

31. Norma Oficial Mexicana NOM-008-SSA3-2010, Para el tratamiento integral del sobrepeso y la obesidad.

32. Rubino F, Marescaux J. Effect of duodenal-jejunal exclusion in a non-obese animal model of type 2 
diabetes: a new perspective for an old disease. Ann Surg. 2004; 239: 1-11.

33. Lee WJ, Wang W, Lee YC, Huang MT, Ser KH, Chen JC. Effect of laparoscopic mini-gastric bypass for type 2 diabetes mellitus: comparison of $\mathrm{BMI}>35$ and $<35$ kg/m2. J Gastrointest Surg. 2008; 12: 945-952.

34. DePaula AL, Macedo AL, Rassi N, Machado CA, Schraibman V, Silva LQ, et al. Laparoscopic treatment of type 2 diabetes mellitus for patients with a body mass index less than 35. Surg Endosc. 2008; 22: 706716.
35. Cohen R. Gastrointestinal surgery for non-obese diabetics type 2. Rev Med Clin Condes. 2009; 20: 698-702.
Correspondencia:
Dr. Luis Guillermo Menchaca Ramos
De la Montaña Núm. 129,
Col. Cumbres, $2^{\circ}$ Sector,
Monterrey, NL, México.
Celular: 8111544416
E-mail: luismenchaca56@hotmail.com 